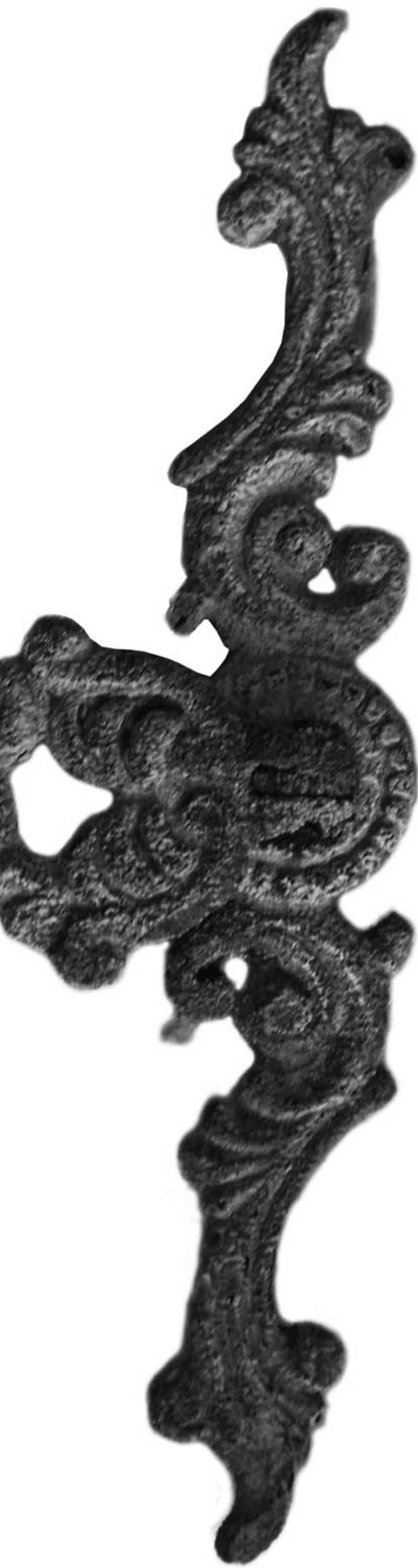

\title{
Entre la arqueología y la restauración. Diálogos con Joaquín Barrio Martín
}

\author{
María del Pilar Tapia López
}

- I doctor Joaquín Barrio Martín no es un profesional del todo común en el ámbito patrimonial iberoamericano: después de licenciarse en arqueología en la Universidad Autónoma de Madrid (UAM), en esa misma ciudad realizó estudios en restauración, en la Escuela Superior de Conservación y Restauración de Bienes Culturales (ESCRBC).

Lejos de debatirse entre la arqueología y la restauración, Barrio ha amalgamado ambas profesiones, y ejerce la restauración arqueológica con la ventaja que le otorga conocer la cultura material desde el punto vista de dos disciplinas que construyen su conocimiento si bien de forma distintiva, complementaria. Como resultado de ello, su actividad profesional lo ha llevado a desarrollar proyectos innovadores que destacan por su perspectiva, contenido y difusión a través de diversas publicaciones y ponencias en congresos a todo lo largo de su natal España, en el resto de Europa y en América Latina. Sorprende la variedad de temas que ha abordado: desde "La revitalización de los ritos de enterramiento y la implantación de la necrópolis de incineración en la cuenca del Manzanares: la necrópolis de Arroyo Butarque (Madrid)" (Blasco, Barrio y Pineda 2007), hasta el "Estudio de los problemas de conservación de los metales arqueológicos dorados provenientes del sitio islámico de Qalat Rabah" (Barrio et al. 2007), es decir, tanto sobre cuestiones arqueológicas como acerca de problemáticas propias de la restauración, lo que ha dotado de gran solidez a su trayectoria.

El crisol de su producción académica es distintivo: aunque en el análisis de materiales utiliza técnicas científicas de punta, como la limpieza láser de metales, e innovaciones tecnológicas para la restauración, no se considera un científico sino un intérprete de resultados, esto es, sus interpretaciones se articulan y materializan con el apoyo de las técnicas y los métodos más adecuados para la restauración de patrimonio cultural arqueológico.

Barrio Martín es, además, un crítico de la esfera profesional de la restauración arqueológica: por un lado lamenta la falta de comunicación entre arqueólogos y restauradores que, en su opinión, suelen "vivir de espaldas" entre sí, y, por el otro, plantea cuestionamientos a aquellos que se dedican a la arqueometría. Su punto de vista es propositivo: reconoce y aplaude el que los científicos 
dedicados a la restauración alcancen un alto grado de especialización.

Como catedrático de arqueología (Conservación y Restauración de Patrimonio Arqueológico) en el Departamento de Prehistoria y Arqueología de la UAM, Barrio sustenta una visión académica; a través de sus opiniones podemos conocer un poco sobre la situación de la enseñanza de la restauración y su reconocimiento profesional en España, sin perder de vista que la UAM no ofrece la licenciatura en restauración, que sólo se imparte como una asignatura dentro de los posgrados (máster universitario y doctorado en arqueología y patrimonio). Sus reflexiones se apoyan, asimismo, en su trabajo como docente en otras universidades, tanto en España como en diversos programas de especialización internacional en América Latina.

Como investigador de la UAM, es fundador y director, desde 2006, del laboratorio Servicio de Conservación, Restauración y Estudios Científicos del Patrimonio Arqueológico (Secyr), coordina el grupo de investigación sobre Tecnología y Conservación de Patrimonio y participa en otros cuerpos académicos enfocados en el estudio, análisis e interpretación de tecnologías, conservación y restauración de materiales arqueológicos. Su ejercicio tiene, entonces, un peso en el desarrollo científico de la restauración en Hispanoamérica.

Su ámbito de influencia no se limita al Viejo Continente: en 2009, al realizarse el Tercer Congreso Latinoamericano de Restauración de Metales en la Escuela Nacional de Conservación, Restauración y Museografía del Instituto Nacional de Antropología e Historia (ENCRyM-INAH, México), dio inicio la relación profesional entre Barrio y el Seminario-Taller de Restauración de Metales de la ENCRyM-INAH, un vínculo que fructificó en el diseño del Curso de Especialización en Patrimonio Cultural Metálico, diplomado en el que participaron docentes de la UAM y la ENCRyM-INAH y que se impartió alternando sedes: durante el verano de 2011, en Madrid, mientras en 2012 en la ENCRyM-INAH. Su sed de ampliar y compartir sus conocimientos se hizo patente a través del compromiso con la formación de especialistas en el área de la conservación y restauración de patrimonio arqueológico tanto dentro como fuera de España. Fue durante la estancia de Barrio en México, y con motivo de esa iniciativa de enseñanza-aprendizaje, cuando tuve la grata oportunidad de entrevistarlo y descubrir su pasión por el patrimonio arqueológico. Esos diálogos abrieron nuevos campos de reflexión sobre el presente y el futuro profesional de la restauración en una comunión hispanoamericana (Figura1).

Pilar TAPIA LóPez (PTL): Doctor Barrio, me gustaría que nos comentara cómo el haberse formado en arqueología y restauración ha impactado su vida profesional, y también cómo fue que originalmente se decidiera a estudiar ambas carreras.

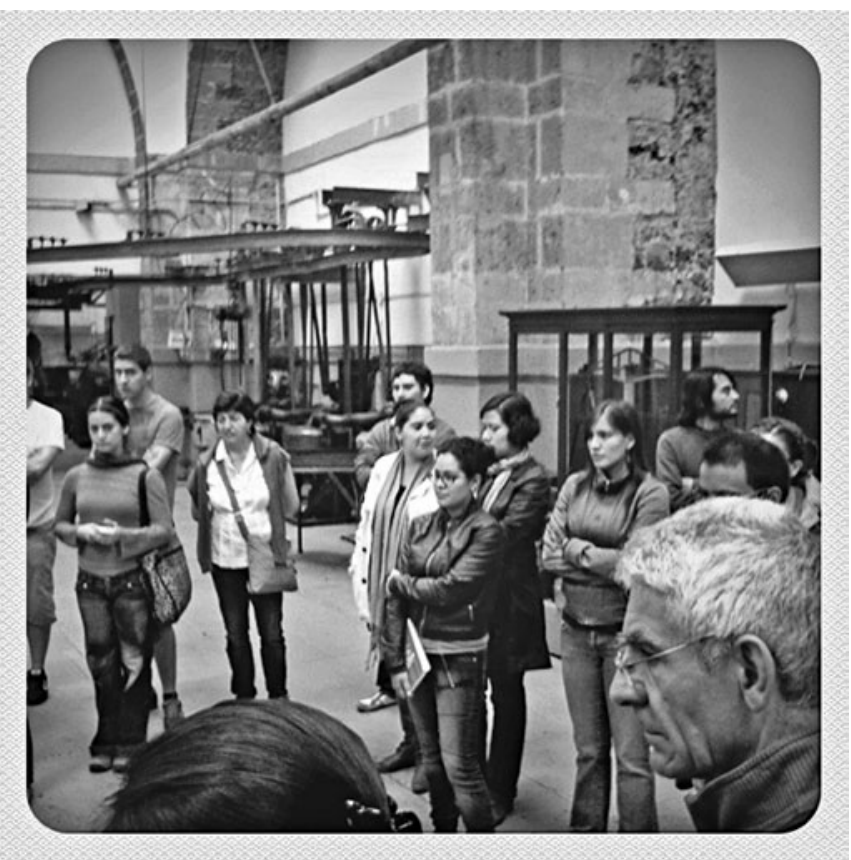

FIGURA 1. Visita a la Casa de Moneda de la Ciudad de México. Diplomado de Especialización en Patrimonio Cultural Metálico, México, ENCRyM-INAH y UAM, 2012 (Fotografía: Gabriel Aguilar, 2012; cortesía: ENCRyM-INAH).

JOAQUín BARRIO MARTín (JBM): Empecé a estudiar arqueología porque siempre sentí esa vocación. Mientras cursaba el tercer año contacté con personas que estaban en la Escuela Superior de Conservación y Restauración de Bienes Culturales y fui con ellos a una excavación de verano; como su trabajo me agradó e interesó mucho, ingresé en dicha escuela en la carrera de restauración arqueológica. Concluí mis cursos de arqueología al quinto año, y el último lo dediqué sólo a estudiar restauración. Obtuve una beca, con la que hice mi tesis doctoral en arqueología prerromana, para posteriormente ingresar como profesor de arqueología, pero al poco tiempo empecé a enseñar también restauración. Nuestra universidad, en ese entonces, era la única que tenía alguna asignatura de restauración dentro de la formación en arqueología, ya que fue fundada por el rector (1973-1978) Gratiniano Nieto, quien también fue creador del Instituto de Conservación y Restauración de Bienes Culturales, ahora Instituto del Patrimonio Cultural de España (IPCE).

Así comencé en la restauración arqueológica, primero como profesor ayudante, después compartiendo parte de las asignaturas de conservación, y finalmente, como titular de todas aquellas relativas a la restauración del patrimonio arqueológico, tanto en el cuarto año de licenciatura en historia como en el máster y doctorado en arqueología y patrimonio en la UAM. Sin embargo, figuro como catedrático de arqueología, y no en restauración, ya que sólo se puede tener cátedra en las áreas de reconocimiento establecidas en el sistema universitario español, en el que aquélla no lo es. Todos los catedráticos 
de restauración en España siguen siéndolo, primero, en pintura, escultura o arqueología, y por el momento soy el único en arqueología sobre restauración de patrimonio arqueológico.

De hecho, siento mayor afinidad por la restauración, pero si requiero impartir arqueología a mis alumnos, lo hago. He hecho investigaciones arqueológicas recientemente y he estado en campañas de arqueología de verano. Me gustan ambas profesiones y procuro compatibilizarlas.

PTL: Nos resulta de gran interés saber si encuentra alguna diferencia en aproximarse a la conservación de los metales arqueológicos como arqueólogo y como restaurador.

JBM: Sólo he encontrado ventajas, ya que la pieza se conoce desde dos perspectivas: la arqueohistórica y la de valor patrimonial. A mi parecer, la única forma de salir adelante es relacionar la arqueología con la restauración. Lo que he podido observar en España y en otras partes del mundo es que son dos disciplinas que han vivido, y viven, una respecto de la otra, "de espaldas". La restauración es poco reconocida y seguimos viendo hoy en día, en España, Europa y otros sitios, a profesionales que no son restauradores tomando decisiones importantes para la conservación de las obras que corresponden al restaurador.

PTL: En México sucede prácticamente lo mismo; ¿por qué cree usted que haya esta dificultad/resistencia para reconocer profesionalmente a la restauración?

JBM: Se debe a la historia de la formación académica en ella. La restauración tiene poco de haber salido de talleres - que eran casi de manualidades-, mientras que la historia del arte o la arqueología existen como disciplinas desde hace más de dos siglos. Sumado a lo anterior, en España hay una escasísima o deficiente estrategia académica. El reconocimiento del máximo estatus tiene que empezar por la formación, no puedes pretender que una profesión que está a nivel de diplomado pueda obtener la máxima calificación. Es una batalla que se puede ganar a través de una excelente formación, y cuando más restauradores sean doctores e ingresen en los máximos estatus académicos - lo que creo que se logrará con el paso del tiempo- la profesión se reivindicará, pero sólo así, por medio de la formación al máximo nivel.

PTL: ¿En España es importante la presencia de un restaurador como parte del personal que debe acudir a una excavación arqueológica?

JBM: Cada vez más se considera la necesidad de la presencia de un restaurador, digamos que es una forma de procedimiento que se ha ido insertando. Sin embargo, el estatus del restaurador en las campañas arqueológicas sigue siendo de segundo nivel: administrativamente, un arqueólogo tiene contrato de nivel uno, mientras que un restaurador lo obtiene de nivel dos, al igual que un arqueólogo-restaurador. Esto, a mi parecer, es ilógico, ya que un arqueólogo-restaurador tiene una formación aún más amplia.

PTL: Considerando, por una parte, que la arqueometría ${ }^{1}$ es una disciplina científica que emplea métodos físicos o químicos para los estudios arqueológicos, y, por la otra, que usted está a cargo del Secyr, me gustaría preguntarle: ¿se considera un arqueómetra?

JBM: No, yo no soy arqueómetra, ni hago arqueometría. No soy físico, ni químico, soy únicamente un usuario de esos métodos científicos de análisis para la conservación. En el Secyr sólo se hacen análisis encaminados a la restauración, nuestra misión es conocer los objetos que vamos a intervenir. Por ejemplo, es interesante saber si un bronce es ternario o binario, pero para su conservación es más importante saber si tiene cloruros o una pátina.

Por otro lado, algunos científicos que se dedican a la arqueometría suelen ser poco cuidadosos respecto de la conservación de un bien cultural, ya que con frecuencia eliminan pátinas y/o cortan objetos con el único fin de realizar un análisis, lo que es intolerable desde el punto de vista de la conservación. Sumado a lo anterior, los datos analíticos obtenidos deben ser interpretados y la mayoría de los arqueómetras no tienen mucha idea de cuál podría ser esa interpretación, más aún cuando se trata de restauración.

PTL: En su opinión, ¿̇e requiere que más restauradores se dediquen a la arqueometría?

JB: Más bien se requiere una arqueometría bien definida en el campo de la restauración; puedes hacer muchas pruebas a una pieza que se va a restaurar, pero de nada sirve sin la adecuada interpretación. Hace falta gente que tenga suficientes conocimientos, para que lo que se pida sea útil. Hay muchas personas que hacen arqueometría sólo por el conocimiento del objeto, lo que es muy loable, ya que ése es el primer sentido de la arqueometría; pero una diseñada para la restauración no tiene por qué tener las mismas condiciones ni los mismos grados de análisis. Lo que hay que hacer es reivindicar la función de cada especialista, lo que no significa limitar las funciones de los demás, sino reconocerlas, así como establecer los derechos y responsabilidades de cada uno.

PTL: ¿Como restaurador trabaja usted exclusivamente metales arqueológicos?

\footnotetext{
${ }^{1}$ El objetivo de la arqueometría es la datación de los objetos y vestigios, así como la caracterización de materiales, del tipo de tecnología empleada y de su origen (o proveniencia).
} 
JBM: No, como restaurador de patrimonio arqueológico es mi responsabilidad y obligación conocer e intervenir otros materiales. De hecho, ahora tenemos un proyecto en el que vamos a intervenir pintura mural arqueológica con láser. En nuestro laboratorio restauramos y estudiamos todo lo que es susceptible de salir en una excavación arqueológica: cerámica, material óseo, marfiles, entre otros (Figura 2).

PTL: Al establecer un proyecto de colaboración académica como el Diplomado de Especialización en Patrimonio Cultural Metálico, ¿por qué eligió a la ENCRyM para desarrollarlo en conjunto con la UAM?

JBM: Porque la ENCRyM es una escuela con prestigio en el mundo iberoamericano; además, su programa académico ha sido consistente, de ella han salido muchos egresados que están en otros sitios y tienen un excelente reconocimiento. También por la seriedad de la gente con la que contacté, con quien vi futuro para este diplomado.

PTL: ¿Cuál es la diferencia entre el diplomado ofrecido en México y el que se impartió en Madrid?

JBM: Fundamentalmente hay dos diferencias: la primera es que en Madrid tuvimos menos alumnos porque la matrícula era costosa, ya que estos estudios deben autofinanciarse; la segunda es que hicimos un diplomado totalmente práctico, lo cual no tenía grandes dificultades por

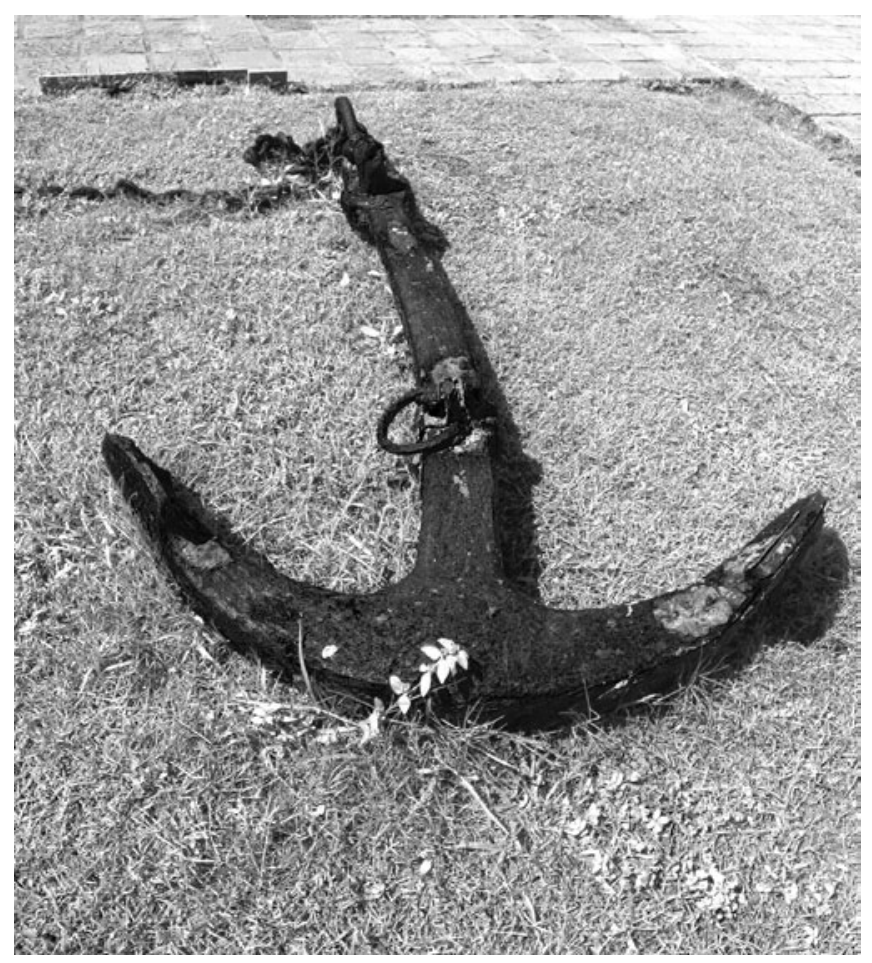

FIGURA 2. Ancla de hierro extraída de contexto subacuático por Apiver y actualmente ubicada en la Fortaleza de San Juan de Ulúa, Veracruz (Fotografía: Ilse Cimadevilla, 2010; cortesía: ENCRyM-INAH). el número de estudiantes inscritos y la cantidad de obra proveniente de nuestras excavaciones que se conservan en la universidad. En la ENCRyM el diplomado tuvo una fuerte carga teórica y el número de alumnos fue mayor. La utilidad de este diplomado es adaptase a las posibilidades y necesidades de cada sitio, de ahí las diferencias.

PTL: Para usted, ¿cuál es la función social del patrimonio arqueológico?

JBM: El patrimonio arqueológico que no cumple con su papel social está dejando de cumplir quizá la mayor función que tiene. Para desempeñarla mejor la restauración es imprescindible, ya que muchas veces un objeto que es una ruina se convierte en algo que un niño verá e interpretará en un museo.

Los bienes arqueológicos se están convirtiendo en un elemento social cada vez más apoyado; basta ver el incremento de los museos de sitio. Dentro de dos, tres o cuatro generaciones, las cuentas que se van a pedir a los especialistas no serán una buena interpretación de investigación en un objeto, sino dónde poder verlo. Lo que se escribe sobre éste podemos cambiarlo o mejorarlo, pero lo determinante es conservar también el elemento material, ésa es nuestra responsabilidad. Eso es lo que hace que, de objetos arqueológicos, pasemos a considerarlos patrimonio arqueológico.

PTL: ¿Podría usted hablarnos sobre su proyecto de la limpieza con láser de bienes metálicos arqueológicos?

JBM: Desde hace muchos años mis proyectos se han enfocado principalmente en la investigación en restauración de metales, porque no hay mucha gente en España que se dedique a esto. En los últimos tiempos nos hemos abocado a investigar la aplicación de la tecnología láser, que está muy experimentada en el mundo de la arquitectura, con beneficios muy importantes.

El láser exige un fuerte costo de inversión, pues hay que comprar el equipo necesario, además de que posteriormente se necesita tiempo para su aprendizaje. Su utilización con los metales es difícil porque las piezas son muy heterogéneas y no se cuenta con cursos específicos para entrenarse. Además, hay que aprender con obra real, ya que las capas de corrosión que te encuentras son irreproducibles (Figura 3).

PTL: A partir de su experiencia, ¿̇odos los objetos metálicos arqueológicos son candidatos a la intervención con láser?

JBM: Todos podrían ser candidatos; sin embargo, algunos ofrecen mejores resultados, y en otros se necesitan mayores esfuerzos en su investigación o cambios en el equipo. La efectividad del láser depende de la pieza, el equipo y el método de aplicación. 


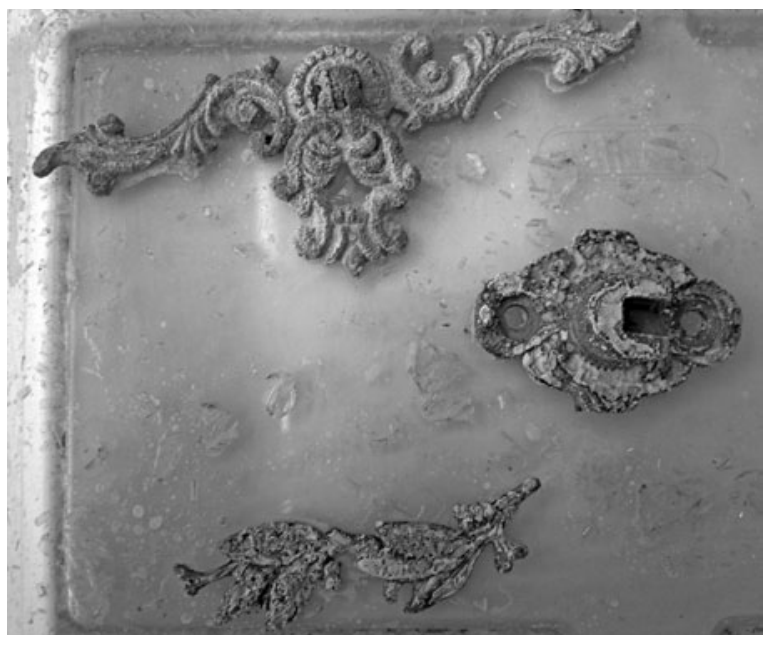

FIGURA 3. Práctica de corrosión. Diplomado de Especialización en Patrimonio Cultural Metálico, México, ENCRyM-INAH y UAM, 2012 (Fotografía: Jannen Contreras Vargas; cortesía: ENCRYM-INAH).

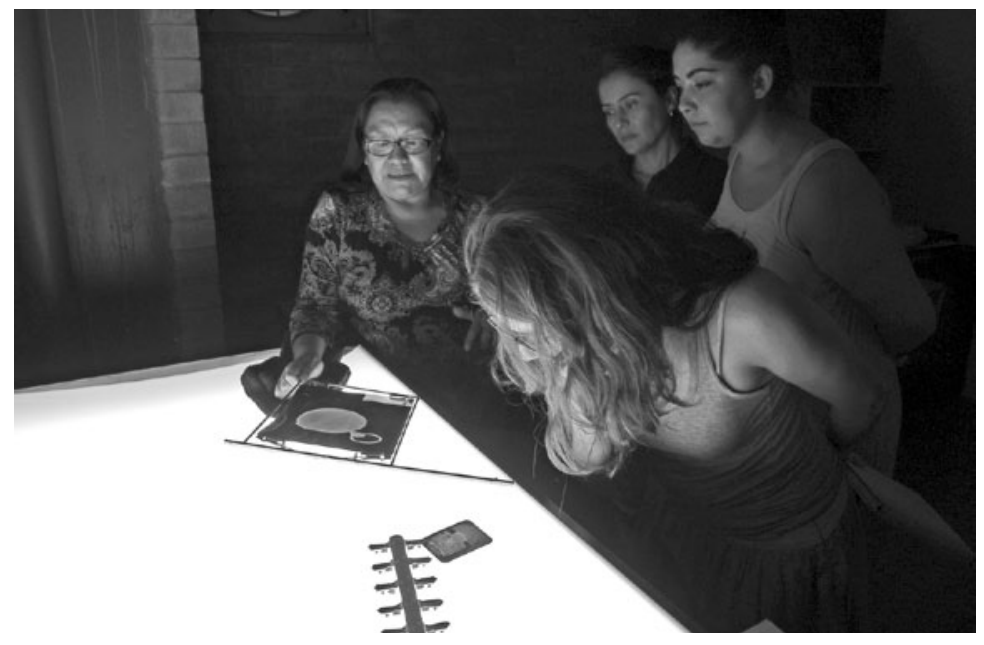

FIGURA 4. Clase sobre radiología con la doctora Josefina Bautista. Diplomado de Especialización en Patrimonio Cultural Metálico, México, ENCRyM-INAH y UAM, 2012 (Fotografía: Jannen Contreras Vargas, 2012; cortesía: ENCRyM-INAH).
PTL: ¿En la actualidad tiene en marcha algún otro proyecto de investigación en restauración arqueológica?

JBM: No, porque no cuento con tiempo. Mi actividad fundamental es como docente, y aunque me gusta mucho la investigación, la satisfacción de un profesor son siempre sus alumnos, el que alguien reconozca tu trabajo como profesor y que además puedas decir: esta persona fue alumna mía, que sea un profesional brillante que adquirió contigo capacidades y lo has podido ayudar, ésa es la mejor retribución a nuestra labor. Restaurar piezas para mí también es importante, y salvar un objeto deja gran gusto, pero constituyen satisfacciones totalmente diferentes. La primera acepta la emoción: uno enseña con cerebro, con corazón y con emociones.

PTL: Desde su punto de vista, ¿cuál es su perspectiva del futuro de la restauración de metales tanto en España como en América Latina?

JBM: Depende fundamentalmente de las posibilidades de financiación de proyectos, ya que se aprende mucho cuando se restaura mucho. Si tenemos muchos proyectos, aprenderemos bastante más. En España hoy la situación económica es mala y prácticamente todos los proyectos de restauración se han cerrado; sin embargo, en América Latina espero que haya mejor financiamiento $y$, por tanto, un gran futuro, por lo que es importante tener buenas instituciones académicas. La gente ya no aprende a restaurar en un taller casero, acude a las instituciones académicas, y eso es lo que dará mayor futuro a nuestra profesión (Figura 4).

La entrevista con el doctor Barrio provoca pensamientos de ida y vuelta sobre la situación de la restauración en México y España que vale la pena destacar.
En una primera instancia, resaltan las similitudes y diferencias. Ciertamente, la situación de reconocimiento y necesidad de profesionales conservadores-restauradores es similar en México y España. Sin embargo, en nuestro país la restauración tiene una gran ventaja frente a otros países; aquí contamos con una licenciatura en restauración que se imparte en la ENCRyM-INAH desde hace ya más de 40 años. Si bien la restauración en México no es todavía suficientemente reconocida como otras profesiones, esto ha cambiado a lo largo del tiempo. Hoy en día se hace más patente que la restauración es una disciplina científica que no trata sólo de "unir fragmentos" o analizar obra desde sus tres instancias: "estética, histórica y funcional", sino que va más allá: al terreno de la interpretación.

Un segundo ámbito es el autorreflexivo. Actualmente la conservación-restauración en México ha caminado hacia un reconocimiento como profesión a escala mundial. No obstante, como lo señala Barrio, queda un camino largo por transitar, que podrá avanzar con pasos cada vez más firmes si los restauradores asumimos el reto de estudiar y conocer otros ámbitos profesionales, participar en encuentros nacionales e internacionales, realizar estudios de posgrado y difundir nuestro quehacer mediante su publicación en libros y artículos en diversos escaparates.

En tercer lugar - la sazón de las obligadas reflexiones en torno de la formación e inserción profesional de los restauradores - resuena una postura única: a pesar de utilizar una amplia gama de recursos científicos y tecnológicos, Barrio no hace análisis sólo porque resulta interesante saber más sobre un objeto: él tiene muy claro que su finalidad no es la arqueometría, sino los análisis científicos que determinan el curso de un proceso de restauración, aquellos cuyo resultado es importante para la toma de decisiones, incluso tal vez algunos que no requieren apegarse tanto a la definición de arqueometría; 
en mi opinión, lo fundamental es que especialistas, como los restauradores, valoren los datos, para confrontar la información obtenida de un análisis científico del objeto y hacer interpretaciones sobre su técnica de factura, uso y funciones, así como de las alteraciones materiales, lo que llevará a la correcta caracterización y valoración de un objeto y, en consecuencia, a su conservación. Por tanto, coincido plenamente con Barrio cuando dice que dentro de 100 años la gente no va a querer leer sólo un buen estudio sobre un objeto; va a querer ver el propio objeto, y por eso la restauración cobra tal importancia. Al reafirmar que el restaurador es un profesional completo, capaz de obtener, rescatar, interpretar, conservar y difundir la información contenida en un bien cultural, Barrio recobra una esencia indiscutible de nuestra profesión.

Por ello sus palabras dejan una visión general de lo que debe ser la restauración: una profesión científica en la que se interpretan datos y se confronta información

\section{Resumen}

Reconocido mundialmente, arqueólogo y restaurador de patrimonio arqueológico, el doctor Joaquín Barrio Martín tiene, como producto de su trabajo, más de una centena de publicaciones donde resulta clara la relación simbiótica entre una y otra ramas de especialización: la arqueología y la restauración. Estos diálogos no sólo son una oportunidad para mostrar la peculiar personalidad de este profesional - a través de opiniones y comentarios es posible comprender por qué es un referente para la investigación y el tratamiento de objetos metálicos- sino también un crisol para reflexionar sobre algunos aspectos de la actualidad y el futuro de la restauración en el ámbito iberoamericano.

\section{Palabras clave}

Joaquín Barrio Martín, metales arqueológicos, conservación de patrimonio arqueológico. con la única finalidad de recuperar la memoria de una cultura, a través de la conservación de los valores contenidos en los objetos creados por ella. Quedan por definirse los ingredientes para esta alquimia.

\section{Referencias}

Barrio, J., J. Chamón, M. Ferretti, M. Arroyo, A. I. Pardo, Aurelio Climent, M. D. Ynsa y Patricia Carolina Gutiérrez 2007 "Study of the conservation problems of the archaeological gilded metals from the Islamic site of Qalat Rabah", en Actas del International Congress Conservation of Metal ICOMCC, Rijmuseum, Ámsterdam, 17-27 de septiembre de 2007, Preprints, vol. I, 10-16.

Blasco, C., J. Barrio y P. Pineda 2007 "La revitalización de los ritos de enterramiento y la implantación de la necrópolis de incineración en la cuenca del Manzanares: La necrópolis de Arroyo Butarque (Madrid)", Zona Arqueológica, 10 (II), 216-238.

\section{Abstract}

Joaquín Barrio Martín, PhD, a world-renowned archaeologist and restorer of archaeological materials, has produced more than one hundred publications, in which the symbiotic relationship between his two professions is made clear. This interview is not only an opportunity to show Barrio's distinctive personality - his opinions and comments make it clear why he is a point of reference for the research and conservation treatment of metal objects - but is also an opportunity to examine the present and future of restoration in Latin America.

\section{Key words}

Joaquín Barrio Martín, archaeological metals, archaeological conservation.

Título en inglés: In-between Archaeology and Restoration. Dialogues with Joaquín Barrio Martín 